\section{Design of a Flexure Rotational Time Base With Varying Inertia}

\author{
E. Thalmann ${ }^{1}$ \\ Instant-Lab, Microcity, \\ Federal Polytechnic School of Lausanne (EPFL), \\ Rue de la Maladière 71b, \\ CH-2000 Neuchâtel, Switzerland \\ e-mail: etienne.thalmann@epfl.ch
}

\section{S. Henein}

Instant-Lab, Microcity,

Federal Polytechnic School of Lausanne (EPFL),

Rue de la Maladière 71b,

CH-2000 Neuchâtel, Switzerland

e-mail: simon.henein@epfl.ch

Flexure oscillators are promising time bases, thanks to their high quality factor and monolithic design compatible with microfabrication. In mechanical watchmaking, they could advantageously replace the traditional balance and hairspring oscillator, leading to improvements in timekeeping accuracy, autonomy, and assembly. As MEMS oscillators, their performance can rival that of the well-established quartz oscillator. However, their inherent nonlinear elastic behavior can introduce a variation of their frequency with amplitude called isochronism defect, a major obstacle to accurate timekeeping in mechanical watches. Previous research has focused on addressing this issue by controlling the elastic properties of flexure oscillators. Yet, these oscillators exhibit other amplitude-related frequency variations caused by changes of inertia with amplitude. In this article, we not only improve existing models by taking into account inertia effects but also present a new way of using them to adjust the isochronism defect. This results in a better understanding of flexure oscillators and an alternative way of tuning isochronism by acting on inertia instead of stiffness. This also opens the door to promising architectures such as the new rotation-dilation coupled oscillator $(R D C O)$ whose symmetry has the advantage of minimizing the influence of linear accelerations on its frequency (the other major limitation of flexure oscillators). We derive analytical models for the isochronism of this oscillator, show a dimensioning with compensating inertia and stiffness variations, and present a practical method for postfabrication isochronism tuning by displacing masses. The models are validated by finite element method (FEM) and mockups serve as preliminary proof-of-concept. [DOI: 10.1115/1.4050558]

Keywords: compliant mechanisms, mechanical oscillators, isochronism, design of innovative devices design of machine elements, spring design

\section{Introduction}

1.1 Flexure Mechanical Watch Time Bases. The Swiss watchmaking industry, with an annual export of about 20 billion Swiss francs, is the country's third largest exporter and accounts for approximately $1.5 \%$ of its gross domestic product $[1] .{ }^{2}$ Mechanical watches constitute about $80 \%$ of these exports (in value) and rely substantially on innovation pushing the limits of mechanical

\footnotetext{
${ }^{1}$ Corresponding author.

${ }^{2}$ More information is available on Switzerland's official website: www.eda.admin ch/aboutswitzerland/en/home/wirtschaft/taetigkeitsgebiete/uhrenindustrie.html

Contributed by the Design Innovation and Devices of ASME for publication in the Journal of Mechanical Design. Manuscript received August 18, 2020; final manuscript received March 5, 2021; published online May 14, 2021. Assoc. Editor: David Myszka.
}

timekeepers to maintain their position. However, despite concerted efforts by the industry, the accuracy of the best mechanical watches seems to have reached a plateau of a few seconds per day. The general consensus in horology is that the quality factor of the oscillator, a dimensionless number that characterizes its damping, needs to be improved for the accuracy to increase [2-4]. Yet, the friction in the bearing of the traditional mechanical watch oscillator, the balance and hairspring (Fig. 1), drastically limits its quality factor. ${ }^{3}$ This fact, together with the recent spreading in the watchmaking industry of high-technology materials and techniques such as silicon components manufactured by deep reactive ion etching [7-9], has motivated the search for new time bases $[10,11]$. Flexure oscillators are a promising replacement for the balance and hairspring since their motion is only guided by the deformation of elastic elements, thus eliminating contact friction $[12,13]$, paving the way to a significant increase in quality factor and a new level of timekeeping accuracy. ${ }^{4}$ Additionally, increasing the quality factor reduces power consumption, leading to greater watch autonomy [11].

The introduction of flexure oscillators in mechanical watches comes with new challenges that must be overcome before they can be implemented. First, the nonlinear elastic behavior of flexures introduces a dependence of oscillation period on amplitude, i.e., an isochronism defect. Second, the deviation of flexures from the motion of ideal linkages can result in changes in the mass distribution of the time base as it oscillates. This causes an isochronism defect (through amplitude-dependent inertia variation) and a contribution of gravity to the effective stiffness of the oscillator (and hence an effect on its period). These directly affect timekeeping accuracy. Other external factors can also affect the period such as temperature, magnetic fields, and shocks but they can be addressed with existing materials and techniques [15].

Flexure oscillators also have applications as MEMS time bases for electronic devices, where their frequency stability and potential for miniaturization and batch production can compete with the well-established quartz oscillator $[16,17]$. The main challenge for MEMS time bases is, as for mechanical watch oscillators, to have a stable frequency regardless of external perturbations, except that the main sources of perturbation are different. For instance, isochronism defect is not a significant issue for MEMS oscillators due to their small amplitudes whereas temperature is $[18,19]$. For this reason, this paper focuses on mechanical watch applications. Note that linear accelerations (such as gravity) are important sources of perturbation for both applications, hence the symmetry of the architectures presented here also has potential for MEMS time bases. ${ }^{5}$

1.2 State-of-the-Art. In our previous research on flexure oscillators, we developed design principles that allow to minimize gravity effects $[15,21]$ and introduced the concept of isochronism tuning by changing second-order stiffness properties of the oscillator [22]. We showed that we could compensate isochronism defects by adjusting the stiffness of flexures whose motion is of second order of the main oscillation, that is, the same order as the isochronism defect, see Eq. (6). The fact that this is done by changing oscillator properties without affecting any other crucial characteristic of the pivot (such as nominal stiffness, gravity sensitivity, and parasitic center shift) is called intrinsic isochronism tuning [22] and is considered superior to existing solutions. Indeed, the first flexure pivot oscillator to be introduced in a watch, the 2014 "Genequand System" $[10,11]$, needed an external mechanism to tune its isochronism [23]; other oscillators based on the crossed flexure pivot [24]

\footnotetext{
${ }^{3}$ Air friction also has a significant influence but eliminating it only increases the quality factor by about $50 \%$ : it was increased from 300 to 450 for a balance and hairspring in $99.8 \%$ vacuum [6].

${ }^{4}$ Monolithic fabrication using materials with minimal internal friction such as monocrystalline silicon also contributes to increasing the quality factor [14].

${ }^{5}$ For instance, Ref. [19] presents a kilohertz MEMS flexure time base using a symmetrized tuning fork which is known to minimize the effect of linear accelerations [20]. The megahertz MEMS oscillator in Ref. [18] also presents a similar symmetry.
} 


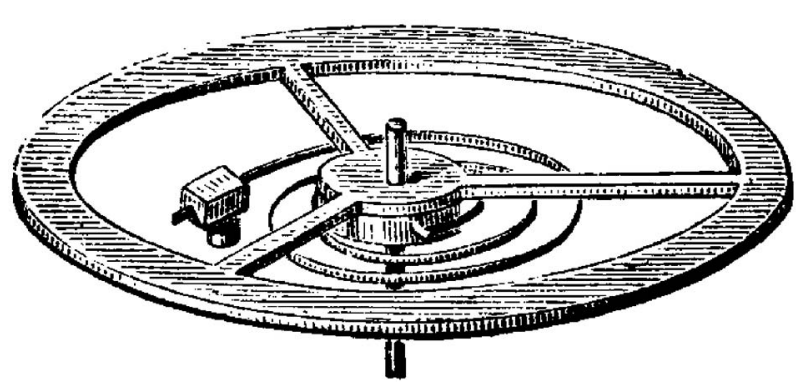

Fig. 1 Classical mechanical watch balance and hairspring oscillator [5]

use special values for the point at which the leaf springs cross and the angle between them to reach isochronism [21,25-28], which, however, does not qualify as isochronism tuning since changing these parameters affects other crucial properties of the pivot $[21,22,29,30]$.

1.3 New Concept of Inertial Isochronism Tuning. In previous research, we noticed that the deviation of flexures from the motion of ideal linkages resulted in changes in the mass distribution of our time bases as they oscillate, thus affecting their isochronism. For instance, the gravity insensitive flexure pivot (GIFP) oscillator $[15,21]$ has a parasitic tilt of its rotation axis and the "co-RCC" oscillator $[15,22,31]$ has an inward motion of its intermediate bodies due to the parasitic center shift of the two remote center of compliance (RCC) flexure pivots it comprises. These effects were neglected, assuming that they were small and could be compensated by acting on the restoring torque nonlinearity (i.e., stiffness variation) of the flexures [22]. Similar inertia variations are also present in other existing flexure time bases [32-34].

In the present article, we show how to take into account the effect of inertia variations in our previous isochronism model. This has several advantages. First, this completes the analysis of the isochronism of flexure time bases and allows to take into account inertia effects into the design instead of compensating them a posteriori. Second, this widens the spectrum of potential new flexure time bases by including architectures with significant inertia variations. For instance, we introduce the new rotation-dilation coupled oscillator (RDCO) family (Fig. 2) whose symmetry has the advantage of inherently minimizing the influence of linear accelerations on oscillation frequency, the other major obstacle to the implementation of flexure time bases [20,21]. Third, we present an alternative way of tuning isochronism, by acting on the inertia variation of the oscillator instead of its stiffness variation. This concept is new and is the main contribution of this article. As a result, new practical methods of tuning isochronism post-fabrication emerge that are potentially simpler than the ones based on the stiffness $[10,22]$. For instance, displacing masses or removing mass by laser ablation [35] is a much less sensitive operation than removing matter from slender flexures to affect their stiffness, as we suggested in Ref. [22]. Some of these results were announced in the first author's $\mathrm{PhD}$ thesis [15].

Remark 1. It is well known that inertia variations can be used to tune the nominal frequency of rotational oscillators. This is for example implemented on balance and hairspring oscillators by changing the position of inertia blocks or screws placed on the balance [37,38]. Inertia variations with temperature have also been used to minimize the effect of temperature on frequency, for example, with bimetallic balances [37, pp. 259-262]. These concepts are however different from the one proposed in this article as they result in a modified inertia that is not a function of oscillation amplitude and therefore does not affect isochronism.

Remark 2. Our concept is analogous to the Huygens' 1657 theoretical isochronism corrector for the pendulum depicted in Fig. 3 $[39,40]$ : he proposed to compensate the isochronism defect resulting from the nonlinear relation between gravity restoring torque and angular position of the pendulum by replacing the rod with a flexible cord that unwinds off a cycloid. This essentially corresponds to changing the active length $L$ of the pendulum as it swings. The analogy with changing the inertia $J$ of a rotational oscillator as it rotates can be seen from the formulas for angular frequency, namely, $\sqrt{k / J}$ for a harmonic rotational oscillator of stiffness $k$ and $\sqrt{g / L}$ for an ideal pendulum under gravity $g$. Pushing the analogy further, tuning the isochronism defect by acting on the inertia variation would correspond to tuning the curvature of Huygens' cycloidal cheeks.

1.4 Structure of the Paper. Section 2 presents the new RDCO family and describes its kinematics. Examples of flexure implementations are given and the design is validated qualitatively on mockups. In Sec. 3, we derive analytical models for the inertia variation and stiffness variation of the RDCO and describe their effect on its isochronism. In Sec. 4, we validate the concepts of this paper by designing a flexure implementation where the inertia and stiffness variations compensate each other to reach theoretical isochronism. We show that the isochronism of the RDCO can be tuned by controlling either its stiffness variation (Sec. 4.2.1) or its inertia variation (Sec. 4.2.2).

\section{Design and Kinematics}

Figure 2 shows a kinematic diagram of the RDCO. The mechanism consists of $n \geqslant 3$ inertial bodies linked to each other by sliders (prismatic joints) to form a loop ( $n=3$ in Fig. 2). Each rigid body is linked to the ground by a connecting rod with a pivot at both extremities, one connected to the ground (pivot $A$ ) and the other to the inertial body (pivot $B$ ). The joints are placed such that they have rotational symmetry of order $n$ with respect to the center $O$ of the system. An extra degree-of-freedom (DOF) is added by allowing a pivoting motion in one of the sliders, see Fig. 2. The sole purpose of this extra DOF is to avoid overconstraining the system; it is not activated during the motion of the system. Grübler's formula for planar linkages [41] gives a mobility $M=1$ for the RDCO in Fig. 2 with $N=8$ bodies and $j=10$ joints with each a $\operatorname{DOF} f_{i}=1$ :

$$
M=\sum_{i=1}^{j} f_{i}-3(j-N+1)=10-3(10-8+1)=1
$$

Knowing that there are no internal DOFs, this shows that the 1-DOF motion depicted in Fig. 2 is obtained without overconstraints (for a planar mechanism).

2.1 Flexure Implementation. For this system to act as a mechanical oscillator, spring components must be added. This happens naturally in the flexure implementation. Any combination of flexures presenting equivalent properties to the ideal kinematic

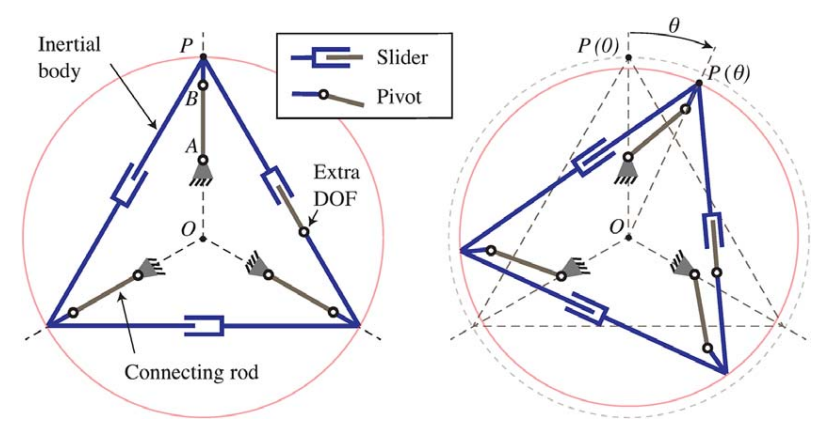

Fig. 2 Kinematic diagram of the RDCO using ideal joints in nominal position (left) and rotated (right) [36] 


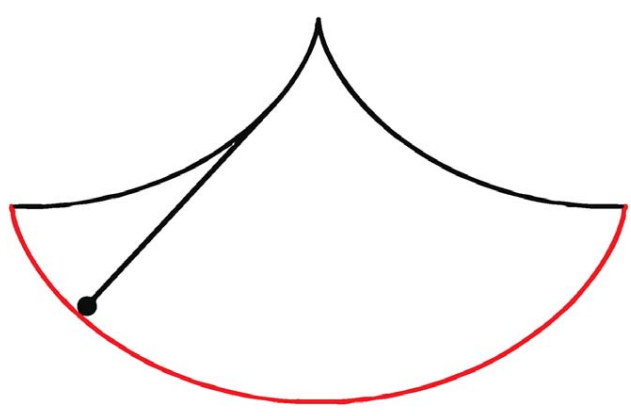

Fig. 3 Huygens' isochronism correction for the pendulum with cycloidal cheeks changing its active length as it oscillates

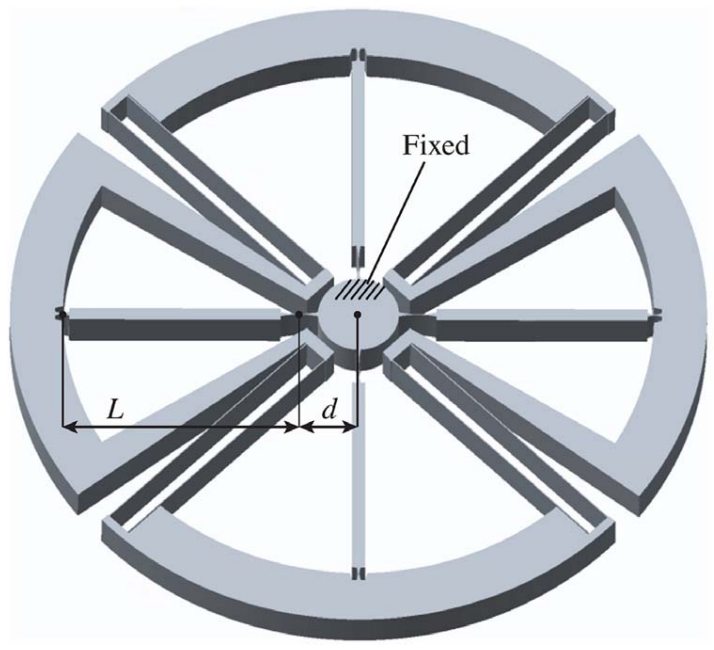

Fig. 4 Example of RDCO physical implementation diagram of Fig. 2 can be used. Figure 4 shows an example where truncated circular notch flexure hinges [13, Section 3.5.10] are used for pivots $A$ and $B$ and parallel leaf springs are used for the sliders. Parallel leaf springs actually have a parabolic motion but it is assumed that they closely approximate a linear motion for the small deformations considered here [13, Section 4.1]. In this implementation, two orthogonal axes of symmetry were chosen instead of a rotational symmetry (so as to have symmetric functions with respect to equilibrium) but the design still corresponds to the ideal kinematic diagram of Fig. 2 with $n=4$ inertial bodies. More examples of flexure implementation are given in Sec. 2.3 and Ref. [15, Section 3.3]. Note that when our designs can be monolithically fabricated, i.e., do not need assembly, the extra DOF is omitted, assuming that there is enough flexibility in the system to release the overconstraint.

2.2 Rotation-Dilation Coupling. The kinematic behavior of the system is determined by the dimensionless ratio $\delta=d / L$, where $d$ is the distance from the center $O$ of the system to the axis of pivot $A$ and $L$ is the distance from the axis of pivot $A$ to the axis of pivot $B$, see Fig. 4 and Table 1. The signs of $d$ and $L$ are defined with respect to the direction from $O$ to $B$. We classify the RDCO architectures according to three domains of the parameter $\delta$, summarized in Table 1 .

(a) When $\delta>0, d$ and $L$ are of same sign. The connecting rods are connected to the ground between the inertial loop and point $O$.

(b) When $-1<\delta<0, d$ and $L$ are of opposite sign and $d<L$. The connecting rods cross each other inside the inertial loop.

(c) When $\delta<-1, d$ and $L$ are of opposite sign and $d>L$. The connecting rods are connected to the ground outside of the inertial loop.

Figure 2 shows the motion of the system as it is displaced from its equilibrium position. We call this motion rotation-dilation coupling,

Table 1 Influence of parameter $\delta$ on the kinematics of the RDCO

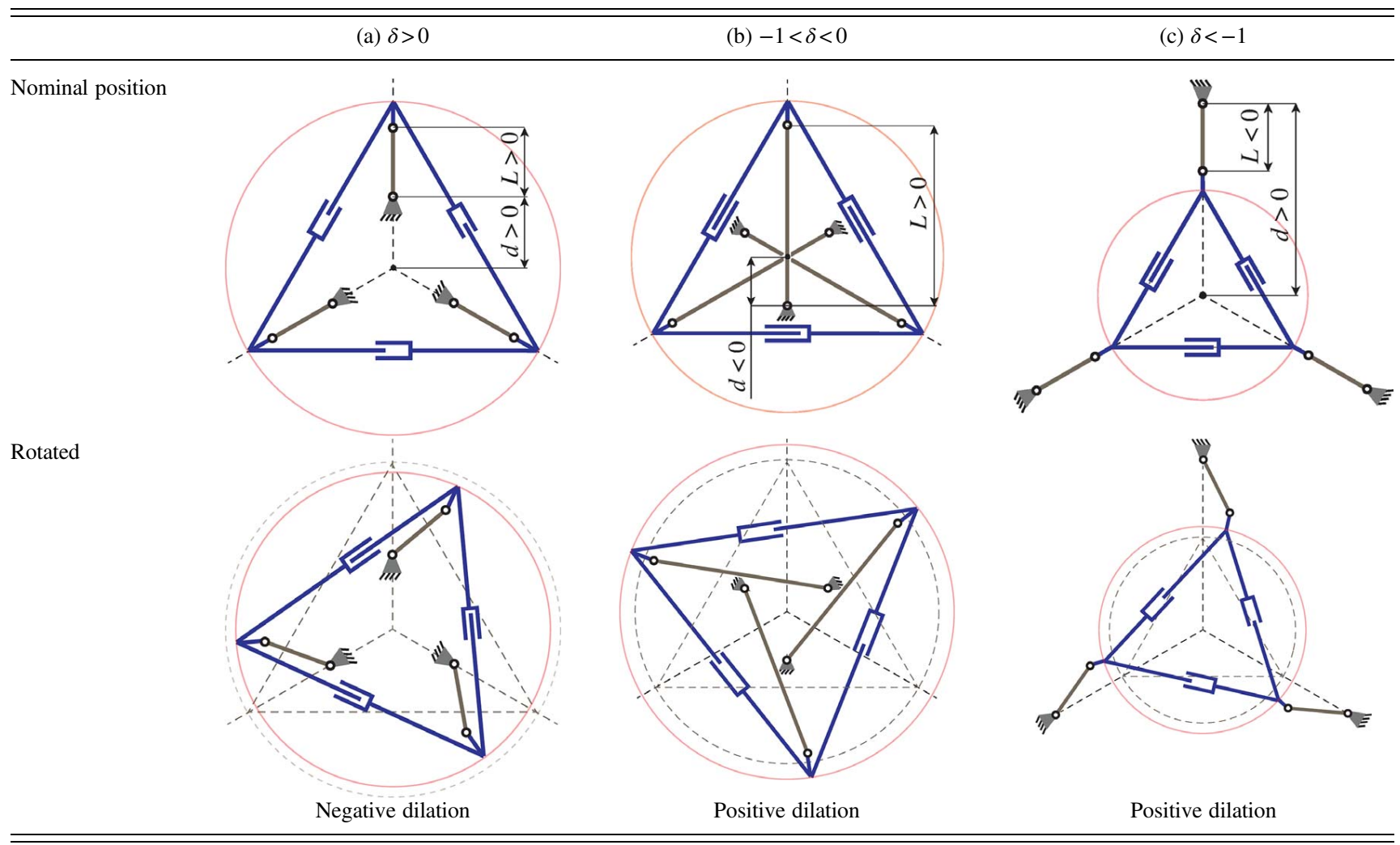




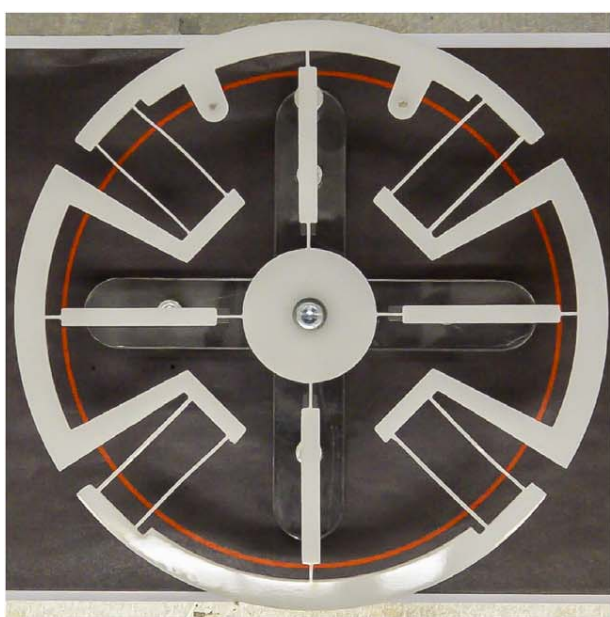

(a)

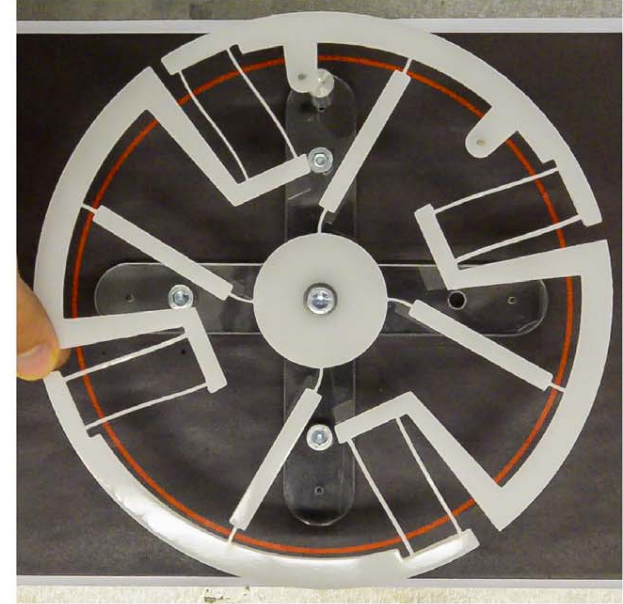

(b)

Fig. 5 Mockup of the RDCO with $\delta>0$ and $n=4$ inertial bodies in (a) nominal position and (b) rotated, showing a negative dilation. Video is available [43].

where the rotation is defined by the angle $\theta$ swept by a vector from the center $O$ to a point $P$ on the inertial body and the dilation is defined by the change of length of this vector. As shown in Table 1 and Eq. (8), the parameter $\delta$ influences this dilation:

- When $\delta>0,\|\overrightarrow{O P(\theta)}\|<\|\overrightarrow{O P(0)}\|$ and the inertial bodies move towards each other. We call it a negative dilation.

- When $\delta<0,\|\overrightarrow{O P(\theta)}\|>\|\overrightarrow{O P(0)}\|$ and the inertial bodies move away from each other. We call it a positive dilation.

2.2.1 Particular Case: $\delta=0$. When $\delta=0$, the connecting rods are attached to the ground at the center of the system and the size of the system stays constant as it rotates (no dilation).

2.2.2 Particular Case: $\delta=-1$. When $\delta=-1, d$ and $L$ are of opposite sign and go to infinity. It is noted that circular motion becomes a straight line motion when the radius goes to infinity. This motion can be implemented by replacing pivot $A$ by sliders with axis tangential to a circle with center $O$ [15, Fig. 3.16].

2.3 Qualitative Design Validation. Mockups were built to validate qualitatively the kinematics of the RDCO. The

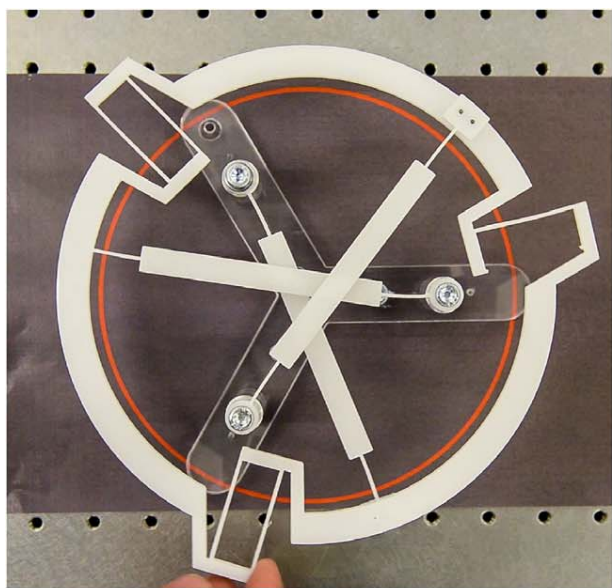

(a) configurations (a) and (b) of Table 1 are shown in Figs. 5 and 6, respectively. In these implementations, rectangular notch flexure hinges $[13,42]$ have been used for the pivots and parallel leaf springs for the sliders. The mockups were fabricated by laser cutting a $5 \mathrm{~mm}$ thick polyoxymethylene sheet. They have an outer diameter of $200 \mathrm{~mm}$ and an admissible angular stroke of approximately \pm 18 deg for configuration (a) and \pm 25 deg for configuration (b). This hardware showed that the system behaves qualitatively as predicted: the system has one DOF and a motion of the inertial bodies closely approximating a rotation about point $O$ coupled to a dilation. The variation in diameter of the mockups can be observed relatively to the circle printed in the background. The two systems confirm that the dilation is negative when $\delta>0$ (Fig. 5 and video [43]) and positive when $-1<\delta<0$ (Fig. 6 and video [44]).

Remark 3. Due to its three-dimensional design, the mockup of Fig. 6 had to be assembled in multiple parts. As a result, the connecting rods do not cross perfectly in the center in the nominal position and one of the parallel leaf springs was removed to prevent overconstraining the pivot. This is equivalent to the extra DOF of Fig. 2.

\section{Analytical Model}

The goal of this paper is to use the inertia variation of the oscillator in addition to its stiffness variation to tune its isochronism

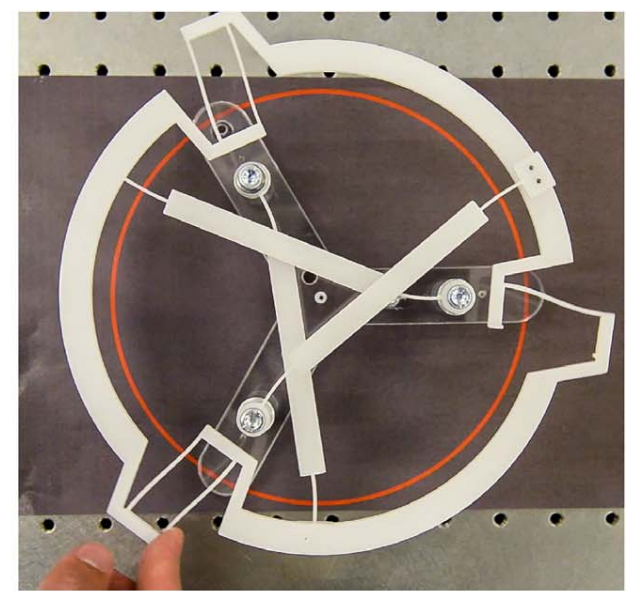

(b)

Fig. 6 Mockup of the RDCO with $-1<\delta<0$ and $n=3$ inertial bodies in (a) nominal position and (b) rotated, showing a positive dilation. Video is available [44]. 
defect. We thus start by deriving an expression for the isochronism defect of a general rotational oscillator with varying stiffness and varying inertia (Sec. 3.1). We then compute these two effects for the RDCO (Secs. 3.2 and 3.3, respectively).

3.1 Isochronism Defect of the Perturbed Rotational Harmonic Oscillator. We consider the case of an oscillator whose restoring torque can be expressed by a power series having only odd terms (assuming the restoring torque to be antisymmetric with respect to equilibrium position)

$$
M=k_{0} \theta+k_{2} \theta^{3}+\mathcal{O}\left(\theta^{5}\right)=k_{0} \theta\left(1+\mu \theta^{2}\right)+\mathcal{O}\left(\theta^{5}\right)
$$

and whose inertia can be expressed by a power series having only even terms (assuming the inertia variation to be symmetric with respect to equilibrium position)

$$
J=J_{0}+J_{2} \theta^{2}+\mathcal{O}\left(\theta^{4}\right)=J_{0}\left(1+\imath \theta^{2}\right)+\mathcal{O}\left(\theta^{4}\right)
$$

Here, $k_{0}$ is the nominal stiffness (i.e., the limiting stiffness as rotation angle goes to zero), $\mu=k_{2} / k_{0}$ is the relative restoring torque nonlinearity [21], $J_{0}$ is the nominal inertia, and $\iota=J_{2} / J_{0}$ is the relative inertia variation.

This oscillator satisfies the differential equation

$$
\ddot{\theta}=-\frac{k_{0}\left(1+\mu \theta^{2}\right)}{J_{0}\left(1+\imath \theta^{2}\right)} \theta+\mathcal{O}\left(\theta^{5}\right)
$$

which, when using again series expansions around $\theta=0$, becomes

$$
\ddot{\theta}=-\frac{k_{0}}{J_{0}} \theta-\frac{k_{0}}{J_{0}}(\mu-\imath) \theta^{3}+\mathcal{O}\left(\theta^{5}\right)
$$

Solving this equation using standard methods of perturbation theory [45, Section (2.3.34)] yields the frequency-amplitude relation of the oscillator

$$
\omega(\Theta)=\omega_{0}\left(1+\frac{3(\mu-\imath)}{8} \Theta^{2}\right)+\mathcal{O}\left(\Theta^{4}\right)
$$

where $\omega_{0}=\sqrt{k_{0} / J_{0}}$ is the nominal frequency as amplitude approaches zero.

Equation (6) gives an explicit expression for the isochronism defect. It shows that isochronism tuning can be performed either by modifying the relative restoring torque nonlinearity $\mu$ by acting on $k_{2}$ (without affecting $k_{0}$ ) or by modifying the relative inertia variation $l$ by acting on $J_{2}$ (without affecting $J_{0}$ ), hence not affecting the nominal frequency $\omega_{0}$. This will be done on the RDCO in Secs. 4.2.1 and 4.2.2, respectively.

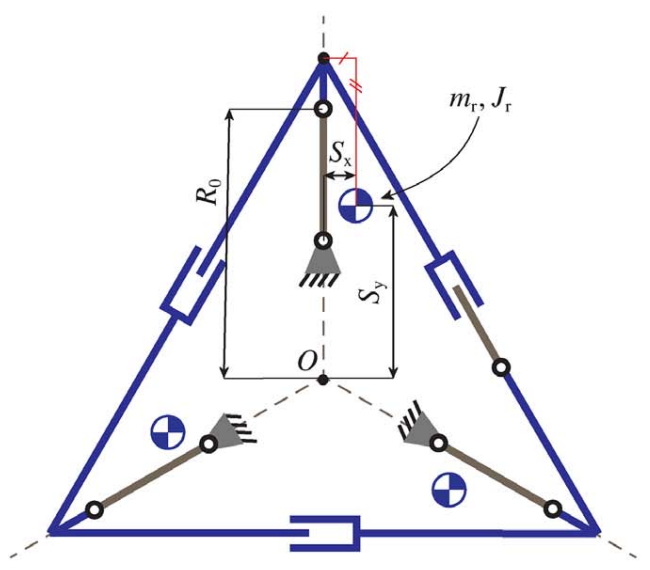

(a)
Remark 4. When the relative restoring torque nonlinearity (i.e., stiffness variation) is equal to the relative inertia variation $(\mu=\imath)$, Eq. (5) becomes the differential equation of the simple rotational harmonic oscillator with constant angular frequency $\omega_{0}$, i.e., with zero isochronism defect.

3.2 Stiffness Variation of the RDCO. The rotational stiffness of the RDCO is calculated with the following assumptions:

- The flexure elements of the RDCO are considered as springs and the other bodies as rigid.

- The system is considered symmetrical: all pivots $A$ have rotational stiffness $k_{A}$, all pivots $B$ have rotational stiffness $k_{B}$, and all the sliders have translational stiffness $k_{t}$.

- The restoring force or restoring torque of the flexure elements can be expressed by series expansion having only odd terms of displacement, see Eq. (2).

- The rotations $\theta$ are small and terms can be expressed using series expansions around $\theta=0$.

- No external force (such as gravity) is acting on the oscillator; we assume that its symmetry minimizes the influence of gravity on its restoring torque [21].

The rotational stiffness of the oscillator is derived from the strain energy of the system with the following steps:

(1) Derive the motion of the two pivots of the connecting rod for a given rotation of the oscillator.

(2) Derive the motion of the sliders for a given rotation of the oscillator.

(3) Express the strain energy of the system for a given rotation of the oscillator.

(4) Compute the rotational stiffness of the system from its total strain energy.

3.2.1 Motion of the Pivots of the Connecting Rod. The angles $\theta_{A}$ and $\theta_{B}$ swept by the pivots of the connecting rod when the system rotates by an angle $\theta$ are obtained by trigonometry, see Fig. 7(b). For small displacements, they can be expressed using series expansions:

$$
\begin{aligned}
& \theta_{B}=\arcsin \frac{d \sin \theta}{L}=\delta \theta+\frac{1}{6} \delta\left(\delta^{2}-1\right) \theta^{3}+\mathcal{O}\left(\theta^{5}\right) \\
& \theta_{A}=\theta+\theta_{B}=(1+\delta) \theta+\frac{1}{6} \delta\left(\delta^{2}-1\right) \theta^{3}+\mathcal{O}\left(\theta^{5}\right)
\end{aligned}
$$

3.2.2 Motion of the Sliders. The motion of the sliders corresponds to the change in distance $q$ between the pivots of the inertial

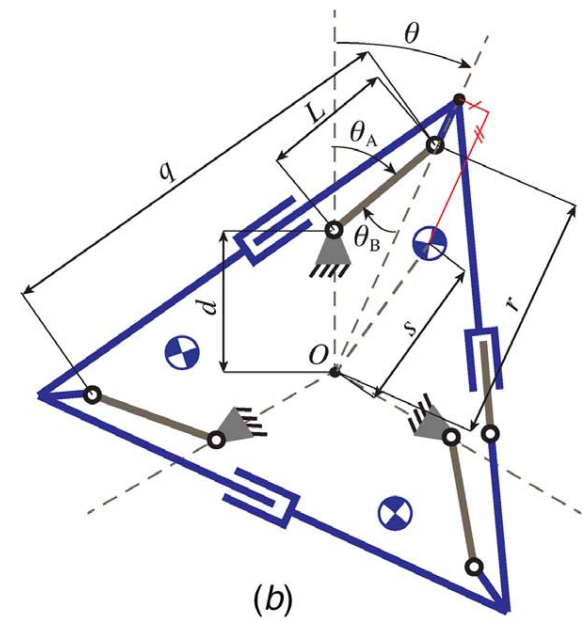

(b)

Fig. 7 Parameters of the RDCO stiffness and inertia models in (a) equilibrium position and (b) rotated by angle $\theta$ 
bodies, see Fig. 7(b). We first derive the distance from the center of the oscillator to these pivots as the oscillator rotates

$$
r(\theta)=d \cos \theta+L \cos \theta_{B}=R_{0}\left(1-\frac{\delta}{2} \theta^{2}\right)+\mathcal{O}\left(\theta^{4}\right)
$$

where $R_{0}=L+d$. We then get the motion of the sliders

$$
\Delta q(\theta)=2 \sin \left(\frac{\pi}{n}\right)(r(\theta)-r(0))=-R_{0} \delta \sin \left(\frac{\pi}{n}\right) \theta^{2}+\mathcal{O}\left(\theta^{4}\right)
$$

3.2.3 Strain Energy. The restoring force of the flexures can be expressed by series expansion:

$$
\begin{gathered}
M_{A}(\theta)=k_{A, 0}\left(1+\mu_{A} \theta^{2}\right) \theta+\mathcal{O}\left(\theta^{5}\right) \\
M_{B}(\theta)=k_{B, 0}\left(1+\mu_{B} \theta^{2}\right) \theta+\mathcal{O}\left(\theta^{5}\right) \\
F_{t}(\Delta q)=k_{t, 0}\left(1+\mu_{t} \Delta q^{2}\right) \Delta q+\mathcal{O}\left(\Delta q^{5}\right)
\end{gathered}
$$

The strain energy of the system for a rotation $\theta$ follows from the sum of the strain energies of each elastic joint

$$
U=n\left(\int_{0}^{\theta_{A}} M_{A}(\nu) \mathrm{d} \nu+\int_{0}^{\theta_{B}} M_{B}(\nu) \mathrm{d} \nu+\int_{0}^{\Delta q} F_{t}(\nu) \mathrm{d} \nu\right)
$$

3.2.4 Rotational Stiffness. Substituting Eqs. (7)-(10) into Eq. (11) and deriving and dividing the strain energy $U$ by $\theta$ yields the rotational stiffness of the RDCO

$$
k_{R}=\frac{\mathrm{d} U}{\mathrm{~d} \theta}=k_{R, 0}\left(1+\mu_{R} \theta^{2}\right)+\mathcal{O}\left(\theta^{4}\right)
$$

where

$$
k_{R, 0}=n\left((\delta+1)^{2} k_{A, 0}+\delta^{2} k_{B, 0}\right)
$$

is the nominal stiffness and

$$
\mu_{R}=\frac{2 \delta(\delta+1)\left(\delta^{2}-1\right) k_{A, 0}+2 \delta^{2}\left(\delta^{2}-1\right) k_{B, 0}+3(\delta+1)^{4} k_{A, 0} \mu_{A}+6 \delta^{2} k_{t, 0} R_{0}^{2} \sin ^{2}(\pi / n)}{3\left((\delta+1)^{2} k_{A, 0}+\delta^{2} k_{B, 0}\right)}
$$

is the relative restoring torque nonlinearity according to Eq. (2). Note that the contribution of the relative restoring torque nonlinearity $\mu_{B}$ of the external pivot is neglected assuming that it is small and that $\delta^{4}$ is small.

Remark 5. We define the rotational stiffness by the secant stiffness, that is the restoring torque divided by the angular displacement $k_{\mathrm{sec}}=M(\theta) / \theta$. This quantity should not be confused with the tangent stiffness, that is the derivative of the restoring torque $k_{\tan }=\mathrm{d} M / \mathrm{d} \theta$.

3.3 Inertia Variation of the Rotation-Dilation Coupled Oscillator. The inertia of the RDCO as it rotates is calculated with the following assumptions:

- All the inertial bodies are the same.

- All the joints are ideal.

- The connecting rods are massless.

- The rotations are small and terms can be expressed using series expansions around $\theta=0$.

The inertia of the RDCO for a rotation $\theta$ is

$$
J(\theta)=n\left(J_{r}+m_{r} s^{2}(\theta)\right)
$$

where $J_{r}$ and $m_{r}$ are, respectively, the inertia and mass of one of $n$ inertial bodies and $s$ is the distance from the center $O$ of the oscillator to the COM of one inertial body (Fig. 7). This distance can be computed as follows:

$$
s^{2}(\theta)=S_{x}^{2}+\left(r(\theta)-\left(R_{0}-S_{y}\right)\right)^{2}=S_{0}^{2}-\delta R_{0} S_{y} \theta^{2}+\mathcal{O}\left(\theta^{4}\right)
$$

where $r$ is given in Eq. (8), $S_{0}=s(0)$ is the distance from $O$ to the $\mathrm{COM}$ of one inertial body at equilibrium, and $S_{y}$ is the projection of that distance against the axis formed by the pivots of the connecting rod (Fig. 7(a)).

Substituting Eq. (16) into Eq. (17) yields the inertia of the RDCO as it rotates

$$
J(\theta)=n\left(J_{r}+m_{r}\left(S_{0}^{2}-\delta R_{0} S_{y} \theta^{2}\right)\right)+\mathcal{O}\left(\theta^{4}\right)
$$

with relative inertia variation according to Eq. (3)

$$
\iota_{R}=\frac{-\delta m_{r} R_{0} S_{y}}{J_{r}+m_{r} S_{0}^{2}}
$$

The results obtained with this model are compared to the ones obtained by the finite element method (FEM) in Fig. 8 and show a good match. The FEM model is described in Sec. 4.1.
Remark 6. The sign of the inertia variation in Eq. (18) depends on the sign of $\delta$, as was already noted through the sign of the dilation in Sec. 2.2.

\section{Example of Implementation and Numerical Validation}

In order to validate the concepts presented in this paper, we design a flexure implementation of the RDCO and show, first, that the inertia and stiffness variation can compensate each other to reach isochronism (Sec. 4.1) and, second, that the isochronism of the RDCO can be tuned by varying either its stiffness variation $\mu$ or its inertia variation $l$ (Sec. 4.2). These results are validated by FEM.

4.1 Design With Compensating Inertia and Stiffness Variation. Two configurations exist where the inertia and restoring torque defects can compensate each other:

(a) $\delta>0$ and the negative dilation of the system $(l<0)$ compensate for the decreasing stiffness of the system $(\mu<0)$.

(b) $\delta<0$ and the positive dilation of the system $(l>0)$ compensate for the increasing stiffness of the system $(\mu>0)$.

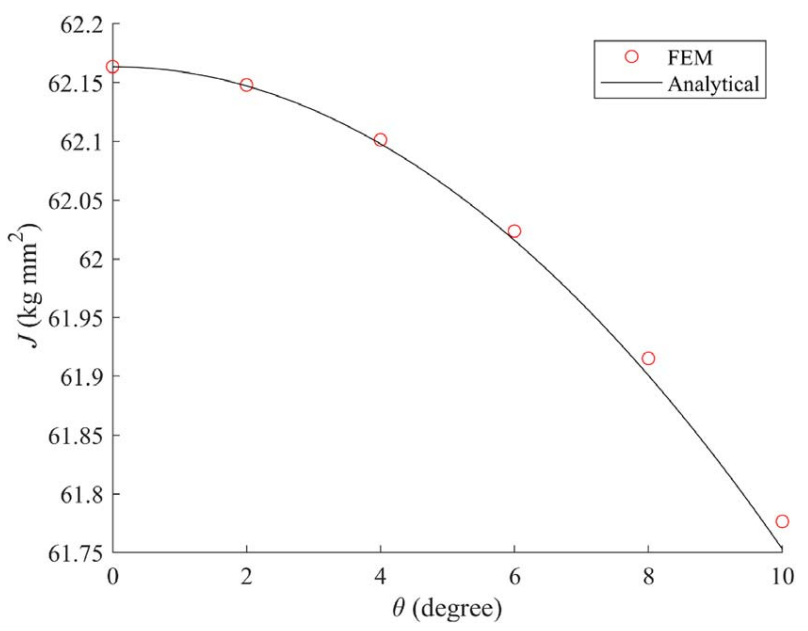

Fig. 8 Inertia $J$ of the RDCO versus rotation angle $\theta$ obtained by FEM and with the analytical model 


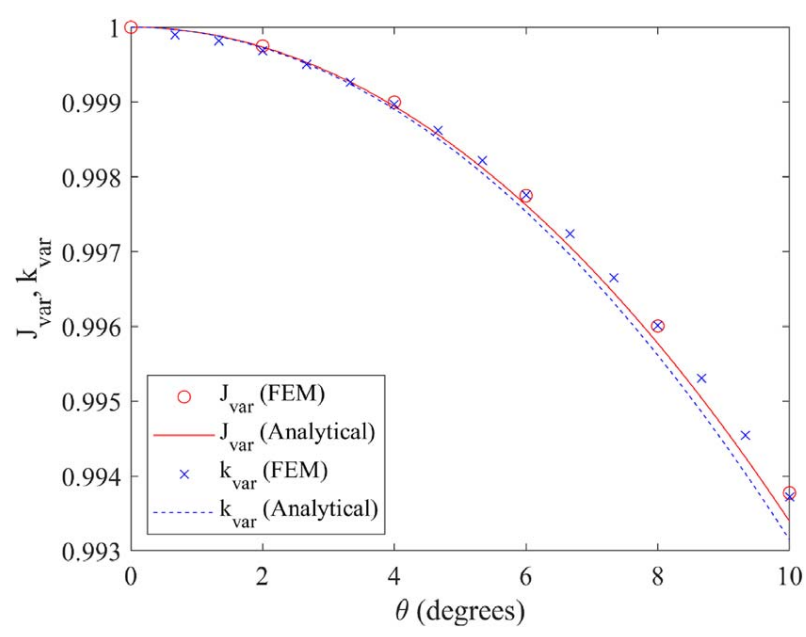

Fig. 9 Inertia and stiffness variation of the example RDCO versus rotation angle $\theta$

We chose to implement configuration (a), which is easier since the connecting rods do not have to cross each other. Our design (Fig. 4) does not target watch specifications but aims at respecting realistic proportions for a titanium prototype of diameter $80 \mathrm{~mm}$ and thickness $5 \mathrm{~mm}$ manufactured by wire electrical discharge machining $(\mathrm{EDM}){ }^{6}$ A relatively large value of $\delta=0.25$ was chosen in order to highlight nonlinear effects. Truncated circular notch flexure hinges were used for pivots $A$ and $B$ with the following dimensions: minimum thickness of $50 \mu \mathrm{m}$ and radius of 10.5 $\mathrm{mm}$ for pivot $A$ and minimum thickness of $150 \mu \mathrm{m}$ and radius of $2 \mathrm{~mm}$ for pivot $B$ (whose deformation is much smaller). Parallel leaf springs were used for the sliders, with a thickness of $130 \mu \mathrm{m}$ and a length of $32 \mathrm{~mm}$. These dimensions can typically be reached by wire EDM [46, Chapter 7].

Figure 9 shows that the inertia and stiffness variation of this oscillator obtained by FEM overlap, hence compensating each other and allowing to reach theoretical isochronism. These results validate the design and concepts presented in this paper.

The oscillator was simulated using the commercial FEM software ANSYS [47] with a mesh of hexahedral elements of type SOLID186 that is refined on the flexures such that there are three elements across their thickness and five along their height (Fig. 10). The truncated circular notch flexures have 10 elements along their length and the parallel leaf springs 32 . The inertia variation $J_{\mathrm{var}}=J(\theta) / J_{0}$ is obtained directly from the FEM data and the stiffness variation is obtained by calculating the secant stiffness for each data point: $k_{\mathrm{var}}=k_{\mathrm{sec}} / k_{0}=M(\theta) / k_{0} \theta$, see Remark 5 .

Remark 7. In order to satisfy the analytical model's assumption that stiffness and inertia are symmetric functions with respect to the equilibrium position, a flexure implementation with axial symmetry was chosen instead of a rotational symmetry such as shown in Fig. 6.

4.1.1 Numerical Validation of the Analytical Stiffness Model. The analytical restoring torque nonlinearity of the oscillator is calculated by substituting the rotational stiffness of the pivots and translational stiffness of the sliders from Ref. [46, Eq. 4.20 and 5.9] into Eq. (14). We showed in previous research that the nonlinearity $\mu_{A}$ of pivot $A$ cannot be calculated accurately using EulerBernoulli beam theory $[15,21]$. This issue is resolved by assuming that $\mu_{A}=0$ to find a first set of dimensions and then fine-tuning the restoring torque nonlinearity $\mu_{R}$ of the oscillator by changing the

\footnotetext{
${ }^{6}$ Titanium alloy TiAl6V4 with Young's modulus $E=114 \mathrm{GPa}$ was chosen for it machinability and high admissible elastic strain $\varepsilon_{\mathrm{adm}}=\sigma_{\mathrm{adm}} / E=0.44 \% \quad[46$, Table B.14]
}

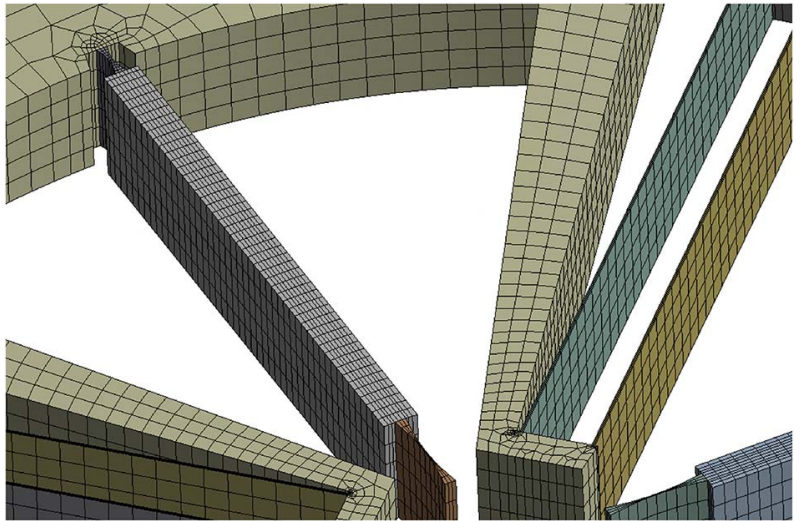

Fig. 10 Close-up view of the mesh of the RDCO finite element model

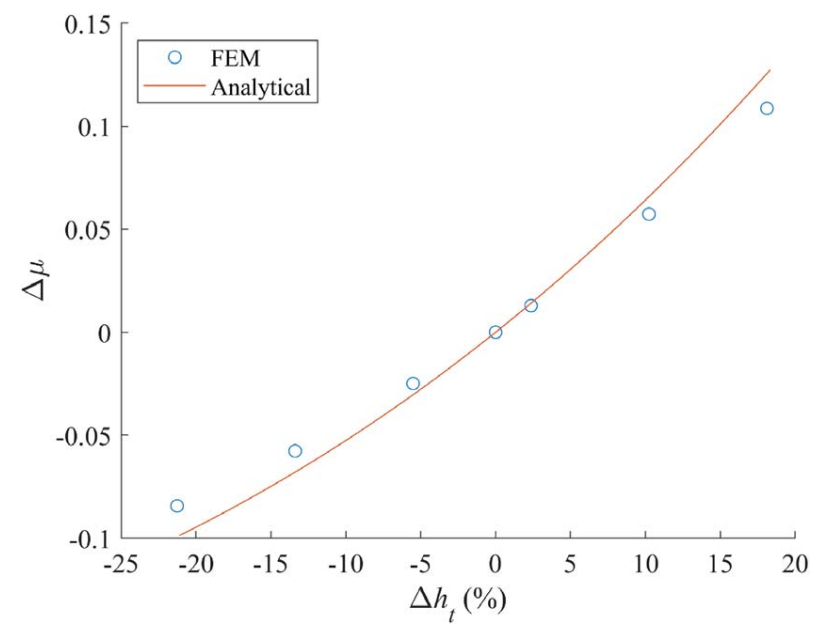

Fig. 11 Analytical and FEM results for the restoring torque nonlinearity tuning (i.e., isochronism tuning) of the RDCO by varying the thickness $h_{t}$ of the parallel leaf springs

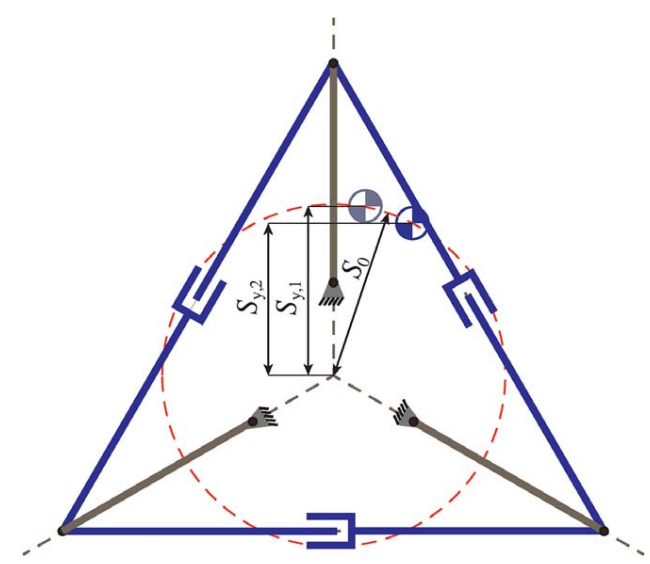

Fig. 12 Tuning of the inertia variation $\imath$ of the RDCO

thickness $h_{t}$ of the parallel leaf springs, as explained in Sec. 4.2.1. For the given implementation, Fig. 9 displays similar analytical and FEM stiffness variations with respective restoring torque nonlinearities $\mu_{R}=-0.225$ and -0.205 . This shows that the analytical model provides an estimation with less than $10 \%$ error despite neglecting $\mu_{A}$. Note that the FEM value of $\mu_{R}$ was obtained by fitting Eq. (2) to the data. 


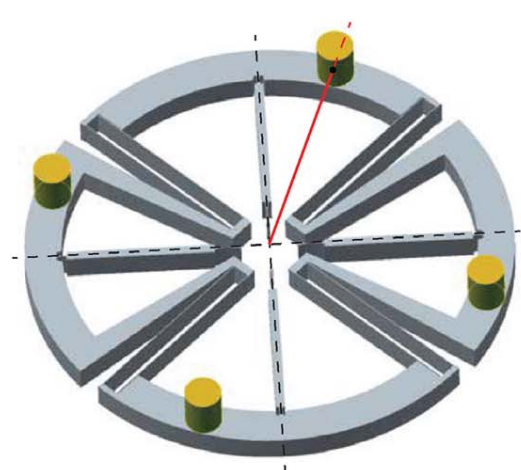

(a)

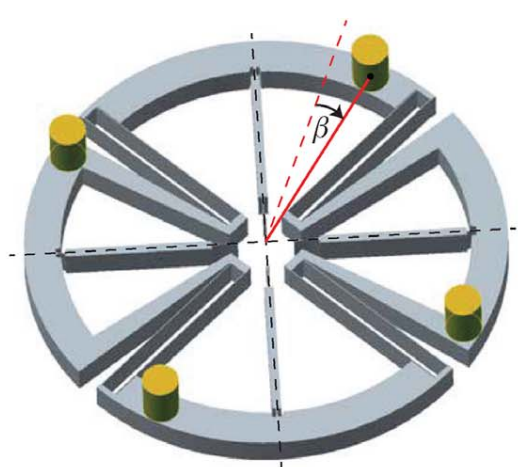

(b)

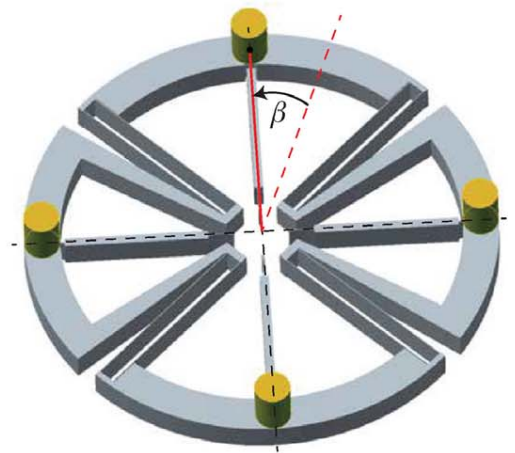

(c)

Fig. 13 Inertia isochronism tuning masses for the RDCO: (a) $\beta=0,(b) \beta=11 \mathrm{deg}$, and (c) $\beta=-20 \mathrm{deg}$

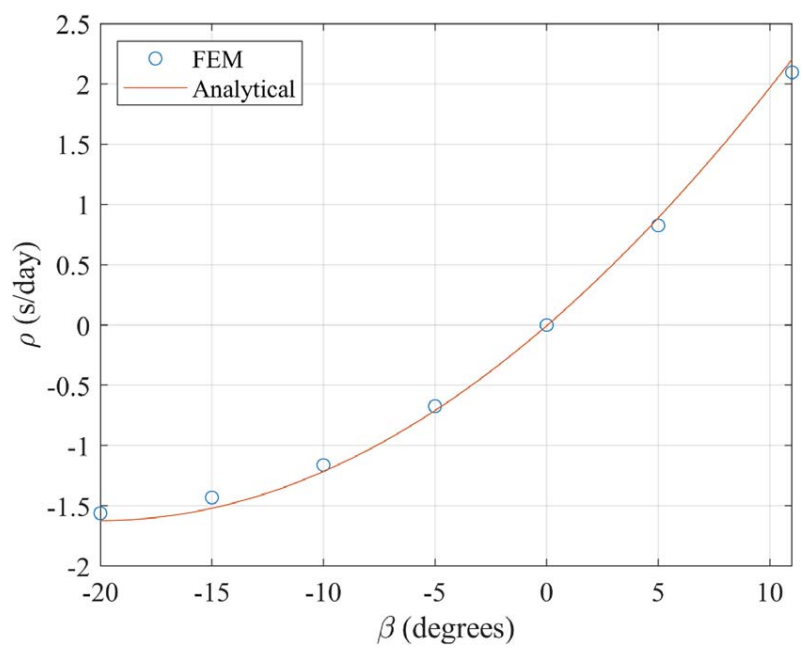

Fig. 14 Isochronism tuning for the RDCO by varying the angular position $\beta$ of tuning masses. Results obtained by FEM and with the analytical model are displayed in terms of daily rate $\rho$ for a $20 \%$ amplitude decrease from reference amplitude $\Theta_{1}=10 \mathrm{deg}$.

4.1.2 Numerical Validation of the Analytical Inertia Model. Since our flexure implementation has two different types of inertial bodies (Fig. 4), we adapted Eq. (18) as follows:

$$
l_{R}=\frac{-\delta R_{0}\left(m_{r, 1} S_{y, 1}+m_{r, 2} S_{y, 2}\right)}{J_{r, 1}+J_{r, 2}+m_{r, 1} S_{0,1}^{2}+m_{r, 2} S_{0,2}^{2}}
$$

where indices 1 and 2 refer to the two different types of inertial bodies. With the mass parameters given in Table 2 , this equation returns an inertia variation $l_{R}=-0.217$ that matches the numerically computed $l_{R}=-0.204$ with less than $10 \%$ error, see Fig. 9 . Note that the FEM value of $l_{R}$ was obtained by fitting Eq. (3) to the data in Fig. 8.

4.2 Isochronism Tuning. Using the models of Secs. 3.2 and 3.3 , we present two ways of tuning the isochronism of the RDCO, by acting either on stiffness or inertia.
4.2.1 Stiffness Isochronism Tuning. The isochronism of the RDCO can be tuned by varying the relative restoring torque nonlinearity $\mu_{R}$ without affecting any other crucial property of the oscillator. As in our previous work [22], this can be done by changing the stiffness $k_{t, 0}$ of flexures that perform a second-order motion of the rotation angle and thus only contribute to the stiffness variation in Eq. (12) without affecting the nominal stiffness $k_{R, 0}$ in Eq. (13). Although the analytical model cannot exactly predict the inertia and stiffness variation of the oscillator, this technique can be used to fine-tune the dimensions obtained using the analytical model to have the two effects accurately compensate each other (Fig. 9).

The tuning calculated with Eq. (14) is compared to FEM results in Fig. 11. Recall that the model cannot accurately predict the absolute restoring torque nonlinearity since $\mu_{A}$ is unknown. The results are hence shown in terms of variation of restoring torque nonlinearity $\Delta \mu$ obtained for a relative thickness variation $\Delta h_{t}$ of the parallel leaf springs. The difference in slope between the analytical and FEM models could be explained by the fact that flexures only approximate the motion of the ideal joints used in the analytical model. It is indeed known that parallel leaf springs actually have a parabolic trajectory and that the rotation axis of notch flexure hinges moves slightly during their deformation [13,42].

Remark 8 . The thickness $h_{t}$ of the parallel leaf springs was chosen as tuning parameter due to its low impact on the design. However, their length $L_{t}$ could have also been used, which might be more suited for post-fabrication fine-tuning [22].

4.2.2 Inertia Isochronism Tuning. The isochronism of the RDCO can be tuned by varying the inertia variation $l_{R}$ without changing any other crucial property of the oscillator. Equation (18) shows that this can be done by changing the parameter $S_{y}$ without changing $J_{r}, m_{r}$, or $S_{0}$, for instance by moving the COM of the inertial part on a circle centered at point $O$ as depicted in Fig. 12. Note that the stiffness properties of the oscillator are not affected by these changes in mass distribution.

Figure 13 shows a practical way of implementing this tuning by moving tuning masses placed on the inertial bodies of the RDCO by an angle $\beta$ on a circle centered at $O$. Note that the nominal position $\beta=0$ is offset with respect to the axis formed by the pivots of the connecting rods such that the tuning can produce either a positive or negative isochronism defect.

In order to provide numerical data for the tuning, we choose a reference amplitude $\Theta_{1}=10 \mathrm{deg}$ and express the isochronism

Table 2 Mass parameters of the RDCO used for the numerical validation

\begin{tabular}{lccccccc}
\hline \hline$R_{0}$ & $S_{0,1}$ & $S_{0,2}$ & $S_{y, 1}$ & $S_{y, 2}$ & $m_{r, 1}$ & $m_{r, 2}$ & $J_{r, 1}$ \\
\hline $34.0 \mathrm{~mm}$ & $34.2 \mathrm{~mm}$ & $30.5 \mathrm{~mm}$ & $34.0 \mathrm{~mm}$ & $30.3 \mathrm{~mm}$ & $11.3 \mathrm{~g}$ & $13.5 \mathrm{~g}$ & $2.29 \mathrm{~kg} \mathrm{~mm}^{2}$ \\
\hline \hline
\end{tabular}


defect in seconds per day for a $20 \%$ amplitude decrease from the $\Theta_{1}$ reference. This is computed by applying Eq. (6) in the definition for daily rate [22, Eq. (7)]. The results are plotted in Fig. 14, showing an effective way of tuning the isochronism of the RDCO with a precision of order $1 \mathrm{~s} /$ day for the chosen amplitude range. Note that the predictions of the analytical model match the numerical result with less than $0.2 \mathrm{~s} /$ day error.

Remark 9. The masses were chosen such that the correction is of order $1 \mathrm{~s}$ /day for the tuning range depicted in Fig. 13. The tuning can be adjusted by changing the mass ratio, inertia ratio, and $\mathrm{COM}$ radius ratio between the mobile and fixed parts of the inertial bodies. In this example, these ratios have the respective values of $0.38,0.007$, and 1.29 for body 2 , see Table 2 .

\section{Conclusion and Future Work}

This article presents a new way of tuning the isochronism of flexure mechanical time bases using their inertia variation, as opposed to the previously known methods that focused on stiffness variations. A simple and practical way of implementing this tuning post-fabrication was devised by displacing masses. A new family of flexure rotational oscillators was introduced that embodies both inertia and stiffness isochronism tuning concepts. Additionally, the symmetry of these architectures has the advantage of addressing another important limitation of flexure time bases, the influence of linear accelerations on their period [15,20-22], making them also interesting candidates for MEMS time bases [16,17].

The concept of inertia isochronism tuning introduced here paves the way for new solutions to this historical problem. The concept is not limited to the RDCO family; it is common for flexure oscillators to have second-order motions resulting in variations of their mass distribution that could be exploited to tune their isochronism. This is, for example, the case with the motion of the intermediate bodies of the "co-RCC" oscillator [22,31] or the parasitic angle of the rotation axis of the "GIFP" oscillator [15,21].

Our search for new time bases has also led to a family of flexure architectures with rotation-dilation kinematics that could find promising applications in other fields. For instance, the RDCO has potential as flexure (also known as "compliant") gripper, where its dilation kinematics offer radial self-centering gripping that can be superior to the conventional two-finger gripping $[48,49]$ while its rotation kinematics facilitate actuation with a standard motor.

\section{Acknowledgment}

We thank Ilan Vardi for his constructive criticism and careful review of the manuscript. We also thank Simón Prêcheur-Llarena and Hubert Schneegans for their assistance in fabricating the mockups of Figs. 5 and 6. Finally, we thank Julia Bierent for her assistance in the dimensioning of the physical implementation example.

\section{Conflict of Interest}

There are no conflicts of interest.

\section{Data Availability Statement}

The datasets generated and supporting the findings of this article are obtained from the corresponding author upon reasonable request.

\section{References}

[1] Federation of the Swiss Watch Industry, 2020, "Exports of Swiss Watches Electronic and Mechanical," www.fhs.swiss/eng/statistics.html
[2] Matthys, R. J., 2004, Accurate Clock Pendulums, Oxford University Press, Oxford.

[3] Bateman, D. A., 1977, "Vibration Theory and Clocks," Horolog. J., 1977-1978 (7 parts).

[4] Vardi, I., 2014, "Le facteur de qualité en horlogerie mécanique," Bull. Soc. Suisse Chronométrie, 75, pp. 57-65.

[5] Privat-Deschanel, P., and Focillon, A., 1880, Dictionnaire Général Des Sciences Théoriques et Appliquées, Delagrave-Garnier, Paris.

[6] Cartier, 2012, "ID Two," Press Release, http://en.worldtempus.com/article/ watches/innovation-and-technology/cartier-id-two-12973.html

[7] Perret, A., Hoogerwerf, A., Niedermann, P., Tang, X.-M., Jeanneret, S., Clerc, P.A., de Rooij, N. F., and Gygax, P., 2002, "Silicon as Material for Mechanical Wristwatches," Symposium on Design, Test, Integration, and Packaging of MEMS/MOEMS 2002, Cannes-Mandelieu, France, Apr. 19, pp. 645-647.

[8] Noell, W., Clerc, P.-A., Jeanneret, S., Hoogerwerf, A., Niedermann, P., Perret, A., and de Rooij, N., 2004, "MEMS for a Watches," 17th IEEE International Conference on Micro Electro Mechanical Systems, Maastricht, The Netherlands, Jan. 25-29, IEEE, p. 39904

[9] Musy, J.-P., Maier, F., and Krüttli, A., 2008, "Echappement et spiral réalisés en Silinvar $^{\circledR}$," Journée d'Etude de la Société Suisse de Chronométrie 2008, La Chaux-de-Fonds, Switzerland, Sept. 17, pp. 51-54.

[10] Barrot, F., Dubochet, O., Henein, S., Genequand, P., Giriens, L., Kjelberg, I., Renevey, P., Schwab, P., and Ganny, F., 2014, "Un nouveau régulateur mécanique pour une réserve de marche exceptionnelle," Journée d'Etude de la Société Suisse de Chronométrie 2014, Lausanne, Switzerland, Sept. 17, pp. 43 48.

[11] Barrot, F., Musy, G., Cosandier, F., Kjelberg, I., Renevey, P., Giriens, L., Schwab, P., Genequand, P., Petremand, Y., Dubochet, O., Ganny, F., and Hamaguchi, T., 2015, "GENEQUAND, a Novel Watch Regulator Based on Compliant Mechanisms," Technical Report.

[12] Eastman, F. S., 1935, Flexure Pivots to Replace Knife Edges and Ball Bearings, an Adaptation of Beam-Column Analysis (Engineering Experiment Station Series), University of Washington, Seattle, WA.

[13] Cosandier, F., Henein, S., Richard, M., and Rubbert, L., 2017, The Art of Flexure Mechanism Design, EPFL Press, Lausanne.

[14] Roszhart, T., 1990, "The Effect of Thermoelastic Internal Friction on the Q of Micromachined Silicon Resonators," IEEE 4th Technical Digest on Solid-State Sensor and Actuator Workshop, Hilton Head, SC, June 4-7.

[15] Thalmann, E., 2020, Flexure Pivot Oscillators for Mechanical Watches, Ph.D. Thesis, EPFL, Lausanne.

[16] van Beek, J. T. M., and Puers, R., 2011, "A Review of MEMS Oscillators for Frequency Reference and Timing Applications," J. Micromech. Microeng., 22(1), p. 013001

[17] Partridge, A., Lee, H., Hagelin, P., and Menon, V., 2013, "We Know that MEMS is Replacing Quartz. But Why? And Why Now?" 2013 Joint European Frequency and Time Forum International Frequency Control Symposium (EFTF/IFC), Prague, Czech Republic, July 21-25, pp. 411-416.

[18] Lee, H., Partridge, A., and Assaderaghi, F., 2012, "Low Jitter and Temperature Stable MEMS Oscillators," 2012 IEEE International Frequency Control Symposium Proceedings, Baltimore, MD, May 21-24, pp. 1-5.

[19] Zaliasl, S., Salvia, J. C., Hill, G. C., Chen, L., Joo, K., Palwai, R., Arumugam, N., Phadke, M., Mukherjee, S., Lee, H., Grosjean, C., Hagelin, P. M., Pamarti, S., Fiez, T. S., Makinwa, K. A. A., Partridge, A., and Menon, V., 2015, "A 3 ppm $1.5 \times 0.8 \mathrm{~mm} 21.0 \mu$ A $32.768 \mathrm{kHz}$ MEMS-Based Oscillator," IEEE J. Solid-State Circuits, 50(1), pp. 291-302.

[20] Hetzel, M., 1962, "Le diapason et son influence sur l'horlogerie," Bull. Ann. Soc. suisse Chronométrie, IV-1962, pp. 666-679.

[21] Kahrobaiyan, M. H., Thalmann, E., Rubbert, L., Vardi, I., and Henein, S., 2018, "Gravity-Insensitive Flexure Pivot Oscillators," ASME J. Mech. Des., 140(7), p. 075002 .

[22] Thalmann, E., Kahrobaiyan, M. H., Vardi, I., and Henein, S., 2020, "Flexure Pivot Oscillator With Intrinsically Tuned Isochronism," ASME J. Mech. Des., 142(7), p. 075001.

[23] Henein, S., and Schwab, P., 2014, "Isochronism Corrector for Clockwork Escapement and Escapement Provided With Such a Corrector," Patent No. US8672536B2.

[24] Wittrick, W., 1948, "The Theory of Symmetrical Crossed Flexure Pivots," Aust. J. Sci. Res. A Phys. Sci., 1, p. 121.

[25] Di Domenico, G., Hinaux, B., Klinger, L., and Helfer, J.-L., 2016, "Timepiece Resonator With Crossed Blades," Patent No. WO2016096677A1.

[26] Di Domenico, G., Léchot, D., Helfer, J.-L., and Winkler, P., 2017, “Timepiece Resonator Mechanism," Patent No. EP3206089A1.

[27] Helfer, J.-L., Di Domenico, G., and Winkler, P., 2017, “Timepiece Resonator Mechanism," Patent No. EP3200029A1.

[28] Thalmann, E., Kahrobaiyan, M. H., and Henein, S., 2018, "Flexure-Pivot Oscillator Restoring Torque Nonlinearity and Isochronism Defect," ASME 2018 International Design Engineering Technical Conferences and Computers and Information in Engineering Conference, Quebec City, Quebec, Canada, Aug. 26-29, Vol. 5A, ASME, p. V05AT07A013.

[29] Wittrick, W., 1951, "The Properties of Crossed Flexure Pivots, and the Influence of the Point at which the Strips Cross," Aeronaut. Quart., 2(Feb), pp. 272-292.

[30] Zhao, H., and Bi, S., 2010, "Accuracy Characteristics of the Generalized Cross-Spring Pivot," Mech. Mach. Theory, 45(10), pp. 1434-1448.

[31] Kahrobaiyan, M. H., Thalmann, E., and Henein, S., 2020, "Flexure Pivot Oscillator Insensitive to Gravity," Patent No. WO2020016131.

[32] von Gunten, S., Gygax, P., and Humair, L., 2015, "Oscillateur Mécanique," Patent No. EP2273323B1. 
[33] Ypma, W. J. B., and Weeke, S. L., 2019, "Mechanical Watch Oscillator," Patent No. WO2019156552A1.

[34] Bayat, D., Pétremand, Y., and Kjelberg, I., 2018, "Timepiece Resonator With Two Balances Arranged to Oscillate in a Single Plane," Patent No. EP3336613A1.

[35] Conus, T., 2015, “Swatch SISTEM51," Journée d'Etude de la Société Suisse de Chronométrie 2015, Lausanne, Switzerland, Sept. 16.

[36] Thalmann, E., and Henein, S., 2019, "Conceptual Design of a Rotational Mechanical Time Base With Varying Inertia," preprint, engrXiv.

[37] Rawlings, A. L., 1993, The Science of Clocks \& Watches, 3rd and revised ed, British Horological Institute, Upton.

[38] Reymondin, C.-A., Monnier, G., Jeanneret, D., and Pelaratti, U., 1999, The Theory of Horology, Swiss Federation of Technical Colleges, Le Locle, Switzerland.

[39] Huygens, C., 2007, "Horologium Oscillatorium [Latin With English Translation by Ian Bruce]," www.17centurymaths.com/contents/huygenscontents. html

[40] Vardi, I., 2015, "Mathematics, the Language of Watchmaking," Watch Around, 20, pp. 90-94.
[41] Grübler, M., 1917, Getriebelehre: Eine Theorie Des Zwanglaufes Und Der Ebenen Mechanismen, Springer-Verlag, Berlin.

[42] Howell, L. L., Magleby, S. P., and Olsen, B. M., 2013, Handbook of Compliant Mechanisms, Wiley, Hoboken, NJ.

[43] Thalmann, E., and Henein, S., 2020, "Rotation-Dilation Coupled Flexure Oscillator With Negative Dilation," [Video File].

[44] Thalmann, E., and Henein, S., 2020, "Rotation-Dilation Coupled Flexure Oscillator With Positive Dilation," [Video File].

[45] Nayfeh, A., and Mook, D., 1995, Nonlinear Oscillations, Wiley Classics Library ed., Wiley, New York.

[46] Henein, S., 2000, Conception des structures articulées à guidages flexibles de haute précision, Ph.D. Thesis, EPFL, Lausanne.

[47] ANSYS, 2018, “ANSYS® Workbench, Release 19.2, ANSYS Workbench User's Guide."

[48] Wang, J.-Y., and Lan, C.-C., 2014, "A Constant-Force Compliant Gripper for Handling Objects of Various Sizes,” ASME J. Mech. Des., 136(7), p. 071008.

[49] Xu, Q., 2017, "Design and Development of a Novel Compliant Gripper With Integrated Position and Grasping/Interaction Force Sensing," IEEE Trans. Automat. Sci. Eng., 14(3), pp. 1415-1428. 